\title{
Utilización de la técnica Nopaloteca para la crianza de Dactylopius coccus Costa bajo condiciones controladas
}

Use of the Nopaloteca technique for the rearing of Dactylopius coccus Costa under controlled conditions

Angel David Hernández Amasifuen ${ }^{1}$, Luis Antonio Matos Lanfranco ${ }^{1}$, Julio AlcidesRojas Chávez ${ }^{1}$, Andy PaoloSanta Cruz Ventura ${ }^{1}$, Anthony Apolinario Cortez Lázaro' ${ }^{1}$, Luis Alberto Huayna Dueñas ${ }^{1}$

\section{RESUMEN}

Objetivo: Analizar la crianza de la "cochinilla" (Dactylopius coccus Costa) bajo condiciones controladas utilizando la técnica de Nopaloteca en la ciudad de Huacho. Métodos: Investigación experimental básica, se evaluó el ciclo biológico de Dactylopius coccus Costa, infestando 150 pencas de Tuna (Opuntia ficus-indica) colgadas en posición inversa con hembras ovipositoras en nidos de gasa. Las pencas fueron perforadas en su base con alambre y se colocaron en tendederos de metal. El ambiente conto con una temperatura de $30^{\circ} \mathrm{C} \pm 2{ }^{\circ} \mathrm{C}$. humedad de $70 \pm 2 \%$ y con 12 horas de luz artificial y 12 horas de oscuridad. Resultados: Se obtuvo 192 "cochinillas" hembras adultas en estado de oviposición a los 66 días por penca; lo que represento un rendimiento de 9,6 g de grana "cochinilla" fresca por penca y se apreció una aceleración en el ciclo biológico dando una producción de "cochinilla" fresca de 1440 Kg. Conclusiones: La crianza de Dactylopius coccus Costa bajo condiciones controladas utilizando la técnica de Nopaloteca aceleró el ciclo biológico obteniendo hembras adultas en periodo de oviposición a los 66 días.

Palabras clave: Dactylopius coccus Costa, nopaloteca, Oviposición.

\section{ABSTRACT}

Objective: To analyze the rearing of the cochineal (Dactylopius coccus Costa) under controlled conditions using the Nopaloteca technique in the city of Huacho. Methods: fBasic experimental investigation, we evaluated the biological cycle of Dactylopius coccus Costa, infesting 150 clones of Tuna (Opuntia ficus-indica) hung in reverse position with ovipositor females in gauze nests. The planks were pierced at their base with wire and placed on metal clotheslines. The ambient temperature was 30 ${ }^{\circ} \mathrm{C} \pm 2{ }^{\circ} \mathrm{C}$. humidity of $70 \pm 2 \%$ and with 12 hours of artificial light and 12 hours of darkness. Results: A total of 192 adult female cochineals were obtained in oviposition at 66 days per penca; which represented a yield of $9.6 \mathrm{~g}$ of fresh cochineal seed per penca and acceleration in the biological cycle was observed, yielding a fresh "cochineal" production of $1440 \mathrm{~kg}$. Conclusions: HThe rearing of Dactylopius coccus Costa under controlled conditions using the technique of Nopaloteca accelerated the biological cycle obtaining adult females in oviposition period at 66 days.

Keywords: Dactylopius coccus Costa, Nopaloteca, Oviposition.

\footnotetext{
${ }^{1}$ Universidad Nacional José Faustino Sánchez Carrión. Huacho, Perú.
} 


\section{INTRODUCCIÓN}

El Dactylopius Coccus comunmente llamado "cochinilla", pertenecen taxonomicamente al orden Hemíptera y a la familia Dactylopiidae, dentro de su género (Dactylopius) existen nueve especies siendo la "cochinilla" la que es mas considerada por su gran valor comercial, ya que ésta presenta una gran diferencia en la calidad del ácido carminico que contiene, en comparación de las otras especies, las cuales a su vez, son consideradas especies silvestres. La cochinilla tiene por habitad los cladodios de las Tunas (Opuntia ficus-indica), pero ademas este insecto parasita mas variedades de plantas correspondientes al género Opuntia. En los cladodios de la Tuna la "cochinilla" se instala para alimentarse, desarrollarse y reproducirse. (Pérez y Becerra, 2001).

La "cochinilla" emplea una alimentacion del tipo fitófaga; es decir, utiliza un estilete bucal por medio de largas trompas para alimentarse de la savia de los cladodios de las tunas y una vez que pierde la conexión con la planta ya no puede volver a adherirse y seguir alimentándose. Este insecto es oriundo de México y de paises andinos como Ecuador, Perú y Bolivia. (Ortega, 2011).

La reproducción de la "cochinilla" se da a cabo en la misma planta y al momento de colocar los huevos de las crías, la "cochinilla" hembra, excreta una sustancia cerosa de color blanco con el fin de cubrir los huevos y protegerlos de las amenazas de su entorno, es decir, lluvia, sol, corrientes de aire y depredadores. Este insecto pasa por diferente etapas morfológicas hasta llegará ser hembra o macho, ya que este insecto presenta dimorfismo sexual. Primero se da la postura de los huevos, lo cual es lenta y después se continua con que los huevecillos se van uniendo y formando una cadena. A los 10 a 20 minutos los huevecillos se abren y del cual emerge el primer estado ninfal, denominado ninfa 1 , este se divide en ninfa migrante y ninfa esablecida. En ninfa migrante el insecto busca un lugar donde establecerse y puede durar de 2 a 3 días. En ninfa establecida el insecto fija su estilete (aparato bucal) a la penca de la Tuna, y es claramente evidenciado por la aparición de numerosos filamentos cerosos, en total la estadia en ninfa 1 puede durar de 3 a 4 semanas y el insecto permanece cubierto por la cera blanca pulveralenta que adquirió en el estado de huevo. Luego esta ninfa 1 sufre una muda, de la cual queda desnuda de toda la cera blanca, y su cuerpo se observa rojo carmín, pero inmediatamente de terminar la muda, aparece entonces la ninfa 2 - Hembra de que de nuevo cuenta con la cera polvosa que la recubre, este periodo dura de 13 a 18 días a veces hasta 25 días. La ninfa permanece fija a la penca de la Tuna, al finalizar esta fase del ciclo ocurre una segunda muda de la cual emerge la hembra adulta, luego van aumentando de tamaño y alcanzan su madurez a los 37 a 47 días. Tambien aumenta su volumen debido a la cópula, que se efectúa a los 3 o 4 días despues de haber mudado. Después de un periodo de 30 a 68 días (periodo de preoviposición), se inicia la oviposición como tal, durando de 28 a 50 días. Después, el insecto muere. La duración del ciclo de las hembras desde la fase de huevo hasta el inicio de oviposicón es de 68 a 106 días. En el caso de los machos, en la ninfa 2- Macho, la ninfa produce abundante cera filamentosa que forma un capullo en cuyo interior muda dando lugar a una serie de transformaciones y a los siguientes estados de prepupa, donde se distinguen cabeza, tórax, abdomen y antenas,; y el estado de pupa de la que emerge el adulto, el cual se distingue con facilidad por sus dos caudas terminales. Este estadio dura de 18 a 22 días aproximadamente. En el macho adulto, se presentan claramente las alas, genitales y antenas bien desarroladas. Su vida como adulto es corta, de 3 a 5 días, realizando vuelos cortos apareándose con varias hembras. El ciclo biológico tiene un duración promedio de 90 días, que puede acortarse o alargarse dependiendo de los factores abióticos. (Ortega, 2011).

El primer exportador de carmin de "cochinilla" a nivel mundial es el Perú, con una participacion del mercado mundial del $90 \%$, siendo Arequipa la principal región productora de "cochinilla" del Perú, seguida por Nazca, y las secundarias son Ayacucho, Apurímac y Huancavelica. (ADEX, 2016).

El Perú es el único país donde se produce a la "cochinilla" a cielo abierto, donde existen valles desérticos con temperaturas constantes de entre 20 y 25 grados en todo el año, sin lluvia ni frío. Pero en las demás zonas del Perú existen cambios de clima que hacen que la producción de "cochinilla" no sea una alternativa viable para los agricultores, ya que la producción a cielo abierto sería destruida por las altas o bajas temperaturas, así como la lluvia; además de que existen otros depredadores naturales 
que pueden acabar con la producción. Los productores no tienen el conocimiento, ni las instalaciones para que su producción pueda procesarse y luego comercializarse tanto para el mercado nacional como el internacional, y aprovechar la demanda que existe del colorante que se obtiene de la "cochinilla" que puede utilizarse en varias industrias como alimentos, medicina, textil y de cosméticos. (Pérez, 2014).

Un ambiente semicontrolado propuesto en México, corresponde a cobertizos con muy poca inversión, construidos con carrizo, adobe, palma o troncos, piso de tierra apisonada, y pueden o no tener paredes; en este último caso, los cobertizos protegen la "cochinilla" parcialmente de la insolación, alta luminosidad, precipitación y vientos, pero no de los enemigos naturales (Llanderal y Campos, 2001).

Otro método es la utilización de microtúneles rústicos con madera o varilla en $\mathrm{V}$ o $\mathrm{U}$ invertida, sobre la cual se sujeta el material que cubre las pencas infestadas, que puede ser plástico cubierto con otros materiales para dar sombra que regule la temperatura; para sostener las pencas se usan soportes laterales de madera y una red de rafia (Méndez, 2001).

La Nopaloteca es un sistema que se caracteriza por colgar las pencas en posición inversa en tendederos y en 3 o 4 estratos. La densidad de pencas puede ser de 60 pencas por metro cuadrado en tres o cuatro estratos y en "paquetes" de dos o tres hileras separados por un pasillo. Los tendederos pueden ser de distinto diseño y de duración permanente o desmontables (de metal o madera). En este método a las pencas se les perfora la base y en el orificio se engancha y se coloca en un tendedero (Llanderal y Campos, 2001).

La "cochinilla" puede ser una alternativa para la diversificación agrícola, ya que tiene variedad de usos en la industria (Pérez, 2014), para que Huacho no dependa de los mismos cultivos para obtener ingresos, ya que una economía es frágil si depende de pocos cultivos, que además son materia prima y dejan poco margen de ganancia. De este modo se pudría llevar acabo la crianza de "cochinilla" bajo condiciones controladas teniendo en cuenta el tiempo de luminosidad (fotoperiodo), temperatura y evitando depredadores naturales, lluvias, vientos fuertes comunes en el Distrito de Huacho que desprenden a las "cochinillas" de las pencas de Tuna.

La presente investigación tuvo como hipótesis: la técnica de Nopaloteca acelera el ciclo biológico de la "cochinilla" para obtener hembras adultas en menos tiempo y como objetivo analizar la crianza de la "cochinilla" (Dactylopius coccus Costa) bajo condiciones controladas utilizando la técnica de Nopaloteca, en la ciudad de Huacho.

\section{MATERIAL Y METODOS}

El trabajo de investigación se realizó desde el 26 de Octubre del 2016 hasta el 31 de diciembre 2016, completando un periodo de 2 meses.

La zona de crianza de la "cochinilla" (Dactylopius coccus Costa) utilizando la técnica Nopaloteca en condiciones controladas estuvo ubicada en un ambiente de $5 \times 4$ metros de una vivienda en la Urbanización Los Cipreses en la ciudad de Huacho, provincia de Huaura, Departamento de Lima. Ubicada a 60 msnm, con una temperatura media anual de $19.1^{\circ} \mathrm{C}$ y alrededor de $8 \mathrm{~mm}$ de precipitaciones.

Se utilizaron barandas de metal donde se mantuvieron a las pencas colgadas por alambres. Las condiciones controladas de la crianza de la "cochinilla" fueron: La iluminación fue mediante la utilización de 4 focos de 150 watts y 3 de 100 watts con un fotoperiodo de 12 horas de luz y 12 horas de oscuridad, la temperatura $\left(30^{\circ} \pm 2^{\circ} \mathrm{C}\right)$ y la humedad $(70 \pm$ $2 \%)$ fueron registrados por un higrotermógrafo Benetech GM1362, se mantuvo la humedad dando un pequeño rocío a las pencas por aspersión una vez al día.

Se recolectaron 150 pencas de Tuna (Opuntia ficus-indica) de las regiones agricolas del Distrito de Barranca en cajas de madera. Las pencas fueron limpiadas y lavadas con mucho cuidado para retirar las espinas y para eliminar otras especies que estuvieron en las pencas. A cada penca se le perforó en su base con alambre y se colgaron en posición inversa en las varandas de metal. (Figura 1).

Se recolectaron $600 \mathrm{~g}$ de "cochinilla" (Dactylopius coccus Costa) hembras maduras 
de regiones agrícolas del distrito de Huarochiri en bolsas plásticas esteriles, con pocas cantidades por bolsa para evitar que estas se aplasten por el peso de ellas.

Para realizar la infestación de las pencas se utilizaron nidos bolsas de gasa de $6 \times 6 \mathrm{~cm}$ en los cuales se introdujeron hembras ovipositoras dando un peso promedio por bolsa de 2 gramos. Se infestaron las 150 pencas, y en cada una de ellas se colocó 2 bolsitas de gasa con las hembras ovipositoras con una espina de tuna en una cara de la penca. (Figura 2).

El periodo de infestacion duro 10 días, despues del cual las bolsitas de gasa se retiraron para que las ninfas establecidas por debajo de ellas alcanzaran su maximo desarrollo. Durante los siguientes días se siguió el desarrollo del ciclo biológico de la "cochinilla" (Dactylopius coccus Costa) se determinó contando los días transcurridos entre la fecha de infestación y las fechas en que se observaron las primeras hembras maduras y machos maduros.

La variable estudiada fue el número de "cochinillas" hembras por penca al ser esta la que produce el ácido carmínico de un gran valor comercial, registrando en la cara de infestación, la cara opuesta, y el total. Se tomó los datos del ciclo biológico y de producción de "cochinilla". Se tomaron cien hembras maduras ovipositoras al azar, se pesaron de manera individual en una balanza de precisión Henkel ${ }^{\circledR}$ modelo BQ1001CS con 0,01 g de precisión para calcular el rendimiento en peso por penca.

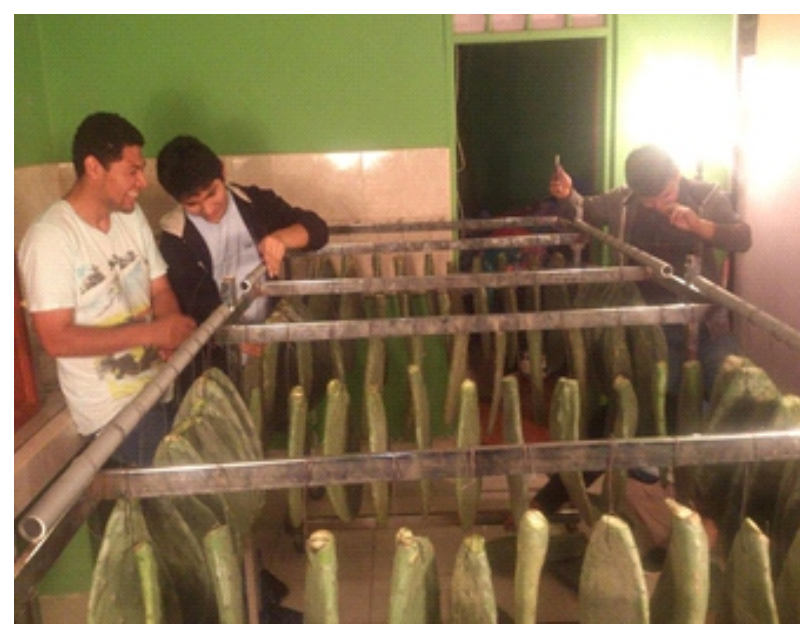

Figura 1. Instalación de las pencas con la técnica Nopaloteca

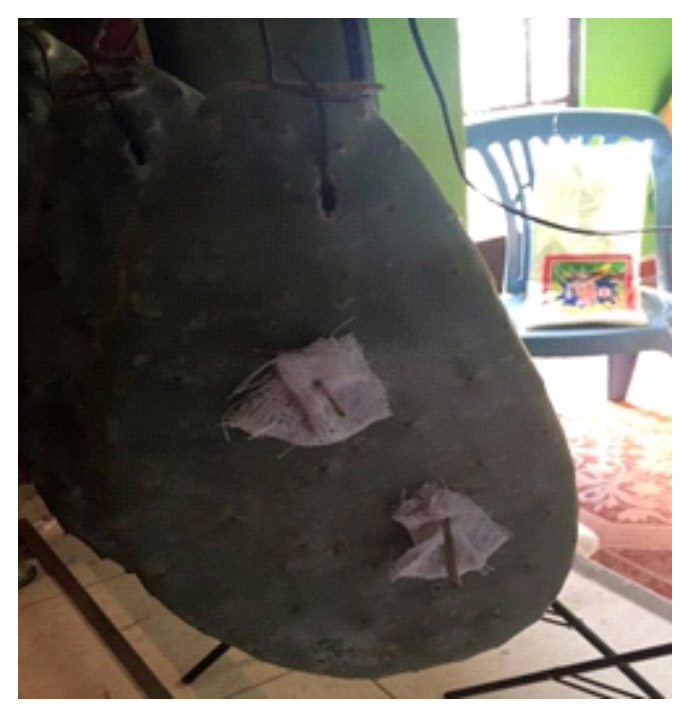

Figura 2. Bolsitas de gasa conteniendo las cochinillas hembras

\section{RESULTADOS}

De las 150 pencas a las cuales se les colocó las bolsas de gasa conteniendo a las "cochinillas" ovipositoras, se logró identificar la presencia de ninfas en 123 pencas al día siguiente de la infestación. Mientras que al segundo día se logró identificar a las ninfas en las 150 pencas. Se apreció a las ninfas de "cochinilla", estas ninfas 1 se hallaban en el primer estado llamada ninfa Migrante, la cual se distingue por un color rojizo y que se desplaza buscando un lugar donde establecerse. (Figura 3).

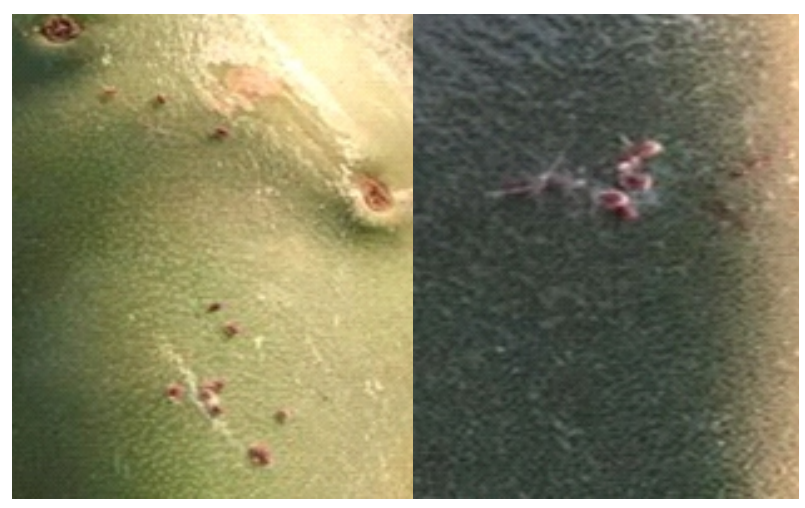

Figura 3. Ninfas 1 en etapa de ninfas Migrantes

Transcurrido 4 días las ninfas 1 mostraron otra etapa de estado ninfal, el cual es denominado ninfa Establecida (Figura 4) la cual fija su estilete en la epidermis de la penca para su alimentación y se evidenció claramente por la presencia de numerosos filamentos cerosos. 


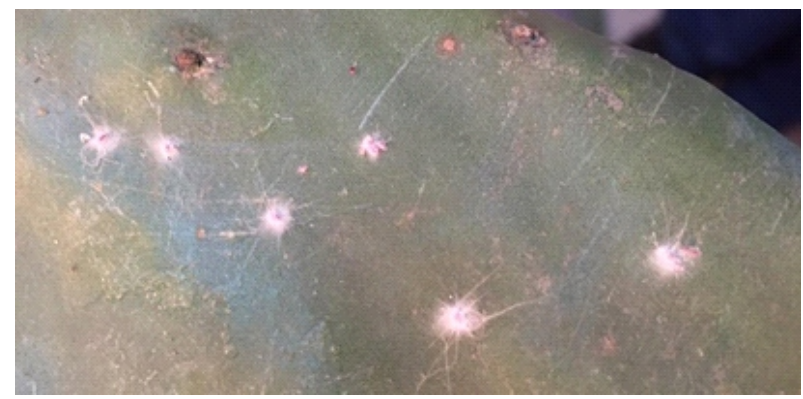

Figura 4. Ninfas 1 en etapa de ninfas establecida, transcurrido 4 días de la infestación

A los 18 días se pudo apreciar como la ninfa 1 sufrió una muda en la cual comenzó a perder su cera blanca pero a las pocas horas de terminada la muda se comenzó a recubrir de cera polvosa dando así lugar a la ninfa 2 (Figura 5 ). Estas ninfas 2 permanecieron fijas aunque muy pocas se desplazaron unos pocos centímetros de su lugar. En los siguientes días se logró observar cómo iban aumentando de tamaño.

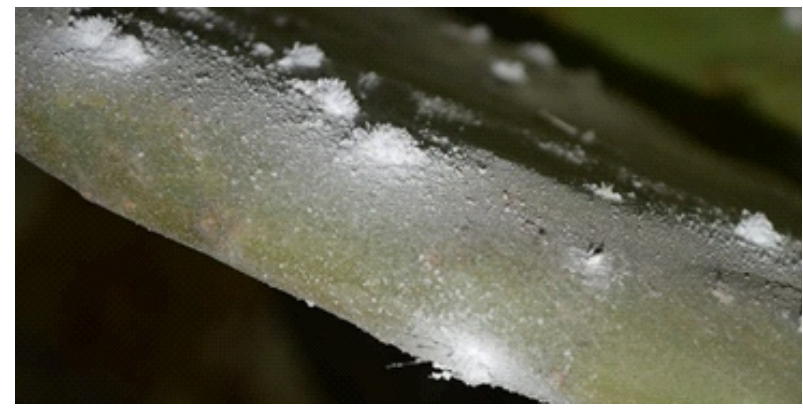

Figura 5. Ninfas 2 cubiertas de cera polvosa, transcurrido 18 días de la infestación

A partir del día 33 las ninfas 2 se comenzaron a diferenciar, algunas comenzaron a dar una segunda muda perdiendo cera polvosa pero en el transcurso de las horas se recubría nuevamente logrando emerger la hembra adulta. Mientras que otras ninfas 2 produjeron abundante cera filamentosa con lo que se formó un capullo, con lo que se diferenció a los machos (Figura 6).

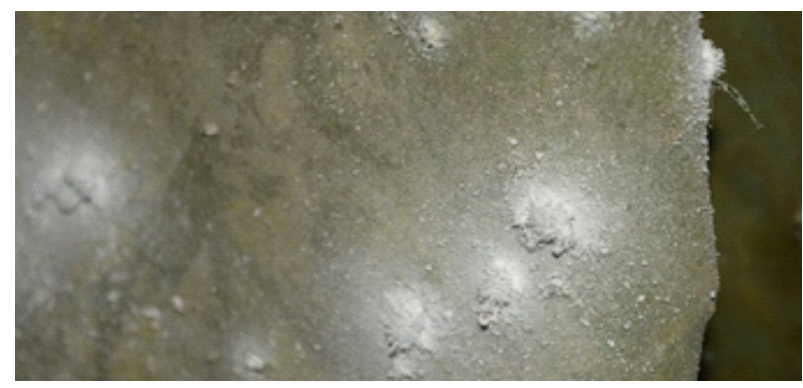

Figura 6. Hembras adultas y capullos, transcurridos 33 días de la infestación
En el día 34 se logró apreciar que el capullo que diferencia a los machos pasaba a su etapa de pre-pupa, 2 días después se distingue el estado de pupa y al día siguiente emergieron los machos adultos, los cuales se distinguieron con facilidad por sus alas (Figura 7).

\section{INTRODUCCIÓN}

Estudios de Salud muestran evidencias de que los niveles elevados de colesterol sérico están asociados con enfermedades cardiovasculares y que el origen del problema era el consumo elevado de colesterol y de grasa saturada. Ante esta situación, la industria de alimentos respondió con un aumento en la producción de aceites vegetales parcial o totalmente hidrogenados (Ballesteros-Vásquez, Valenzuela-Calvillo, Artalejo-Ochoa, RoblesSardin, 2012) dando origen a los ácidos grasos trans (AGT). Estos ácidos grasos trans se forman mediante un proceso de hidrogenación que consiste en la introducción de un gas hidrógeno a los aceites vegetales bajo ciertas condiciones de presión y temperatura usando un metal catalizador (Níquel) (Monrroy, 2009), dicho proceso permite otorgarle al producto características deseables como alargar el tiempo de vida útil, evitar la rancidez, mejorar textura, potenciar sabor y por último abaratar costos de producción. Si bien es cierto presenta múltiples ventajas, también tiene inconvenientes, muchos estudios señalan que consumir en exceso alimentos que contengan (AGT), modifican negativamente el perfil lipídico dado que no solo aumentan los niveles de triglicéridos (TG), Colesterol total (CT), y CLDL sino que reducen el C-HDL, por lo que estaría relacionado con el aumento del riesgo de enfermedades cardiovasculares, hipercolesterolemia, hipertrigliceridemia y arteriosclerosis (Ballesteros-Vásquez, Valenzuela-Calvillo, Artalejo-Ochoa, RoblesSardin, 2012). Estas enfermedades son multifactoriales y resultan de la combinación de causas externas e internas.

Se acepta que los malos hábitos en el estilo de vida son las principales causas en el aumento de este padecimiento, donde la inadecuada alimentación junto al sedentarismo, son los que más repercuten en la salud. La prevención se sustentaría en llevar un determinado régimen de vida que incluya ejercicio físico diario y una dieta equilibrada. Estudios científicos señalan 


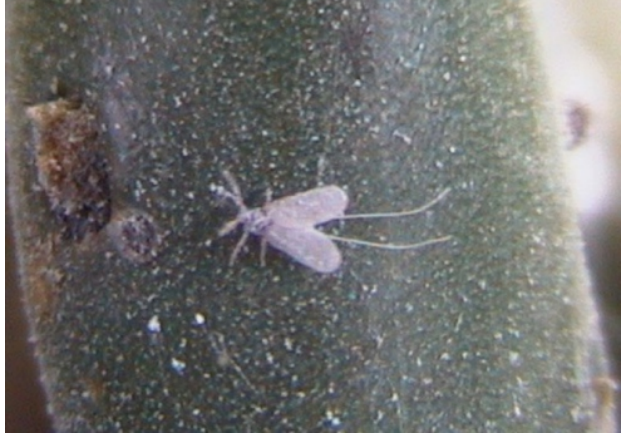

Figura 7. Macho adulto, transcurrido 34 días de la infestación

En el día 66 se logró apreciar el gran aumento de tamaño que sufrieron las hembras debido a la copula y por tanto estas ya se encontraban en periodo de oviposición. (Figura 8)

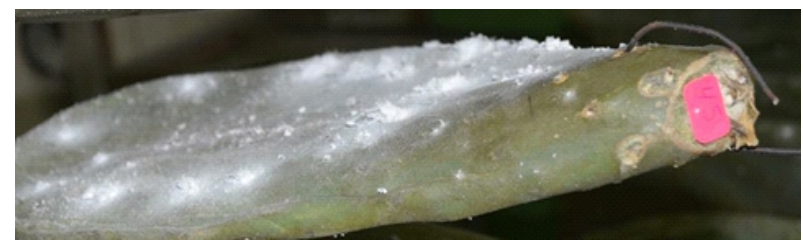

Figura 8. Hembras ovipositoras, transcurrido 66 días de la infestación

La infestación con bolsitas de gasa en pencas colgadas registró $58 \%$ de la población establecida en la cara infestada y $42 \%$ en la cara opuesta (Tabla 1). El promedio de peso de las hembras fue 0,05 $\pm 0,01 \mathrm{~g}$. Este valor al ser multiplicado por el número de hembras por penca da un rendimiento promedio de $9,60 \mathrm{~g}$ de "cochinilla" fresca por penca. Utilizando las 150 pencas la producción de "cochinilla" fresca fue de $1440 \mathrm{Kg}$.

Por lo expuesto anteriormente, el objetivo del presente trabajo fue evaluar la modificación del perfil lipídico sanguíneo en respuesta al ejercicio físico en ratas alimentadas con dietas conteniendo AGT, medido por la concentración de TG, CT, C-HDL y C-LDL.

\section{MATERIAL Y MÉTODOS}

La investigación realizada fue de tipo prospectivo-longitudinal, se llevó a cabo en el Bioterio de la Facultad de Zootecnia de la Universidad Nacional Agraria la Molina y tuvo una duración de ocho semanas. Se trabajó con 24 ratas machos de la especie Holtzman de cuatro meses de edad, con peso promedio de $319 \pm 12,34 \mathrm{~g}$, las cuales recibieron alimento y agua ad libitum durante las ocho semanas que duro el estudio y se les dio exclusivamente el alimento mezclado con margarina que contenía $5,29 \%$ de grasa trans.

El experimento fue dividido en dos etapas: En la primera, denominada etapa de acostumbramiento, las 24 ratas machos se dividieron en dos grupos (dieta estándar y dieta estándar con $2,9 \%$ de AGT) y se colocaron en jaulas metálicas individuales, en las jaulas pares se ubicaron las ratas que consumieron la dieta estándar o dieta que habitualmente consumen las ratas en el Bioterio y en las jaulas impares, las ratas que consumieron la dieta con $2,9 \%$ de AGT, por espacio de una semana. La dieta con $2,9 \%$ de AGT se obtuvo mezclando $1800 \mathrm{~g}$ de la dieta estándar con $200 \mathrm{~g}$ de margarina Primavera en una mezcladora HOBART a velocidad mínima del equipo hasta lograr una mezcla homogénea (ausencia de trozos de margarina). La dieta se preparó cada cinco días y se almacenó en un recipiente oscuro y puesto a refrigeración. A ambas dietas se realizó el análisis de perfil de ácidos grasos.

Finalizada la semana de acostumbramiento los animales fueron pesados y se separaron 6 ratas por cada tipo de dieta según su ganancia de peso para que naden. En la segunda, etapa de entrenamiento de los animales, las ratas seleccionadas para la práctica de ejercicio, fueron puestas a nadar en un cilindro de plástico de 1,10 $\mathrm{m}$ de largo $\times 1 \mathrm{~m}$ de ancho $\mathrm{x}$ $46,5 \mathrm{~cm}$ de altura con un volumen de agua de 320 litros aproximadamente. El grupo de entrenamiento se ejercitó de lunes a viernes por espacio de 40 minutos diarios durante siete semanas. Se registró todos los fines de semana el peso de los animales, así mismo; se

Tabla 1. Número promedio de hembras por penca

\begin{tabular}{rccc}
\hline Cochinilla & $\begin{array}{c}\text { Cara de } \\
\text { infestación }\end{array}$ & $\begin{array}{c}\text { Cara } \\
\text { opuesta }\end{array}$ & Total \\
$\begin{array}{r}\text { Hembras } \\
\text { oviplenas }\end{array}$ & 111 & 81 & 192 \\
\hline
\end{tabular}


Tabla 2. Relación costo-utilidad de la producción de "cochinilla" fresca

\begin{tabular}{ccccccccc}
\hline Cochinilla & $\begin{array}{c}\text { Costo } \\
(\mathbf{S} / .)\end{array}$ & $\begin{array}{c}\text { Capacidad } \\
\text { \# de } \\
\text { Pencas }\end{array}$ & $\begin{array}{c}\text { Producción } \\
\text { penca } \\
(\mathbf{g})\end{array}$ & $\begin{array}{c}\text { Total } \\
(\mathbf{k g})\end{array}$ & $\begin{array}{c}\text { Precio de } \\
\text { venta }\end{array}$ & $\begin{array}{c}\text { Ingreso } \\
(\mathrm{S} / .)\end{array}$ & $\begin{array}{c}\text { Utilidad } \\
(\mathbf{S} / .)\end{array}$ \\
\hline $\begin{array}{c}\text { Método } \\
\text { Nopaloteca }\end{array}$ & 40 & 150 & 9,60 & 1,440 & 80 & 115,20 & 75,20 \\
\hline
\end{tabular}

Tabla 3. Relación costo-utilidad de la producción de "cochinilla" fresca

\begin{tabular}{|c|c|c|c|c|c|c|c|}
\hline \multirow[b]{2}{*}{ Cochinilla } & \multirow[b]{2}{*}{$\begin{array}{l}\text { Costo } \\
\text { (S/.) }\end{array}$} & \multirow[b]{2}{*}{$\begin{array}{c}\text { Capacidad } \\
N^{\circ} \text { de } \\
\text { Pencas }\end{array}$} & \multicolumn{2}{|c|}{ Producción } & \multirow[b]{2}{*}{$\begin{array}{l}\text { Precio de } \\
\text { venta*(S/.) }\end{array}$} & \multirow[b]{2}{*}{$\begin{array}{l}\text { Ingreso } \\
\text { (S/.) }\end{array}$} & \multirow[b]{2}{*}{$\begin{array}{c}\text { Utilidac } \\
\text { (S/.) }\end{array}$} \\
\hline & & & $\begin{array}{l}\text { Por } \\
\text { penca } \\
\text { (g) }\end{array}$ & $\begin{array}{l}\text { Total } \\
\text { (kg) }\end{array}$ & & & \\
\hline $\begin{array}{c}\text { Método } \\
\text { Nopaloteca }\end{array}$ & 250 & 1800 & 9,6 & 17,28 & 80 & 1382,4 & 1132,4 \\
\hline
\end{tabular}

Tabla 4. Relación costo-utilidad de la producción de "cochinilla" fresca de proyección a futuro

\begin{tabular}{|c|c|c|c|c|c|c|c|}
\hline \multirow[b]{2}{*}{ Cochinilla } & \multirow[b]{2}{*}{$\begin{array}{l}\text { Costo } \\
\text { (S/.) }\end{array}$} & \multirow[b]{2}{*}{$\begin{array}{c}\text { Capacidad } \\
\mathbf{N}^{\circ} \text { de } \\
\text { Pencas }\end{array}$} & \multicolumn{2}{|c|}{ Producción } & \multirow[b]{2}{*}{$\begin{array}{l}\text { Precio de } \\
\operatorname{venta}^{*}(\mathrm{~S} / .)\end{array}$} & \multirow[b]{2}{*}{$\begin{array}{l}\text { Ingreso } \\
\text { (S/.) }\end{array}$} & \multirow[b]{2}{*}{$\begin{array}{c}\text { Utilidad } \\
\text { (S/.) }\end{array}$} \\
\hline & & & $\begin{array}{l}\text { Por } \\
\text { penca } \\
\text { (g) }\end{array}$ & $\begin{array}{l}\text { Total } \\
\text { (kg) }\end{array}$ & & & \\
\hline $\begin{array}{c}\text { Método } \\
\text { Nopaloteca }\end{array}$ & 1000 & 7200 & 9,60 & 69,12 & 80 & 5529,60 & 4529,60 \\
\hline
\end{tabular}

${ }^{*}$ Adex 2016

\section{DISCUSIÓN}

La utilización de las bolsitas de gasa fue un método eficiente en la infestación de "cochinilla", la gasa al tener similitudes a las bolsitas de tul lo que coincide con Flores-Flores y Tekelenburg (1995), quienes reportan buenos resultados al utilizar el nido de tul para la infestación de tunas en pie con "cochinilla".

Se apreció que en el ciclo biológico desde huevo hasta llegar al periodo de oviposición en las hembras se dio a los 66 días. Esto puede atribuirse a la alta temperatura a la que se mantuvo a las "cochinillas". Esto concuerda con Méndez (2001) quien explica que a mayor temperatura el ciclo biológico se acorta, pero a temperaturas bajas, se alarga. Con la duración del ciclo biológico registrado se puede obtener hasta tres generaciones de "cochinilla" al año en lugar de 2 o hasta 1 en ambientes abiertos.

Con la infestación con nido de bolsitas de gasa en pencas colgadas registró $58 \%$ de la población establecida en la cara infestada y $42 \%$ en la cara opuesta (Tabla 1). En condiciones de campo se ha observado que la ninfa migrante de primer instar busca las superficies sombreadas de las pencas para establecerse, por lo que se recomienda colocar las bolsas o nidos con las hembras oviplenas en la cara de la penca que no recibe la insolación directa (Condeña, 1997). En esta investigación, el alto porcentaje de infestación en las dos caras de la penca indica que ambas son adecuadas para la fijación de las ninfas, debido a que ninguna de ellas está expuesta a los rayos directos del sol, lo que puede dar una mejor distribución de las ninfas en la superficie total de la penca $y$, por lo tanto, un mejor desarrollo de las mismas.

Se necesitarían 104 pencas de tuna, respectivamente, para producir un kilogramo de "cochinilla" fresca según los resultados obtenidos de nuestra investigación. Este rendimiento supera al reportado por Méndez (2001) de $3 \mathrm{~g}$ de "cochinilla" seca por penca, que equivalen a producir aproximadamente $6 \mathrm{~g}$ de grana "cochinilla" fresca.

Si se considera la relación costo-utilidad de la producción de "cochinilla" fresca de la Tuna (Tabla 2) utilizando el método de Nopaloteca con condiciones controladas genera una buena utilidad al solo necesitar poco gasto económico. Además este método permite una fácil inspección de las pencas durante el manejo y no hay deformaciones de las pencas, tales como desecación, flacidez y muerte de las pencas de Tuna.

Cabe mencionar que en el ambiente utilizado para la crianza de las "cochinillas" sobró mucho 
espacio, por lo que si se aprovecha todo el espacio para colocar más número de pencas y además modificar las barandas para colocar 3 estratos (pisos) tal como proponen Campos y Llanderal (2003) poseer armazones distribuidos en tres niveles para el mantenimiento de las pencas, ya sea colgadas o bien sostenidas mediante una red de rafia. Con esto se podria multiplicar el número de pencas hasta en 12 veces el numero utilizado en este trabajo por lo que si se aplica a una relacion costo-utilidad (Tabla 3 ) los ingresos superarian los S/. 1000.00 nuevos soles.

Teniendo una proyección a futuro se podría aplicar este método de crianza en un ambiente de $16 \times 16$ metros donde se aprovecharía al máximo la capacidad del lugar para colgar las pencas a 3 sustratos con lo que se obtendría mayor cantidad de kilos de "cochinilla" fresca y de esta manera generar una mayor ganancia. (Tabla 4).

\section{REFERENCIAS BIBLIOGRÁFICAS}

ADEX, Perú. (2016). Nota de prensa. D i s pon i ble e n lín e a : http://www.adexperu.org.pe/index.php/p rensa/notas-de-prensa/item/501productores-de-cochinilla-sonafectados-por-precios-especulativos.

Campos, M, \& Llanderal, C. (2003). Producción de grana cochinilla Dactylopius coccus (Homoptera: Dactylopidae) en invernadero. Agrociencia 37(2), 149155.

Condeña, A. (1997). Manejo integral de la tuna y cochinilla para los valles interandinos de la sierra peruana. Universidad Nacional de San Cristóbal de Huamanga. Programa de frutales y elaboración de licores. Ayacucho. Perú, 38-41.

Flores-Flores, V. \& Tekelenburg, A. (1995). Dacti (Dactylopius coccus Costa) dye production. In: Agro-ecology, cultivation and uses of cactus pear. FAO International Technical Cooperation Network on Cactus Pear. Rome. FAO Plant Production and Protection Paper 132, 167-185.
Llanderal C., C. \& M. Campos F. (2001). Sistemas de producción de la grana cochinilla. In: Producción de Grana Cochinilla. Llanderal C., y R. Nieto (eds.). Colegio de Postgraduados. Montecillo, México, 61-67.

Méndez, S. (2001). Cultivo y manejo de la grana cochinilla. In: Cría de la grana cochinilla del nopal para la producción de su pigmento. Llanderal C. C. y R. Nieto H. (eds.). Colegio de Posgraduados. México, 69-77.

Ortega, V. (2011). Comparación del rendimiento del ácido carmínico entre dos procesos de deshidratación de la cochinilla de tunas cultivadas en guano.

Tesis de Grado para la obtención del Título. Escuela Superior Politécnica de Chimborazo. Ecuador.

Pérez, M. (2014). Estudio técnico para la implementación de una planta procesadora de cochinilla para la obtención del carmín. Tesis de Grado para la obtención del Título. Pontificia Universidad Católica del Perú, Lima.

Pérez, M. \& Becerra, R. (2001). Nocheztli: el insecto del rojo carmín; Revista Biodiversitas. 36,1-8.

\section{Correo electrónico:}

kxmcas@hotmail.com

Revisión de pares:

Recibido:

Aceptado: 